\title{
On the Mean Curvature Flow on Graphs with Applications in Image and Manifold Processing
}

\author{
El Chakik Abdallah, Elmoataz Abderrahim, Sadi Ahcene \\ UCBN, GREYC - UMR CNRS 6972, 6.Bvd Marechal Juin, 14050 Caen, FRANCE \\ aelchak@ensicaen.fr, (abderrahim.elmoataz-billah, ahcene.sadi) @unicaen.fr
}

\begin{abstract}
In this paper, we propose an adaptation and transcription of the mean curvature level set equation on a general discrete domain (weighted graphs with arbitrary topology). We introduce the perimeters on graph using difference operators and define the curvature as the first variation of these perimeters. Our proposed approach of mean curvature unifies both local and non local notions of mean curvature on Euclidean domains. Furthermore, it allows the extension to the processing of manifolds and data which can be represented by graphs.
\end{abstract}

\section{Introduction}

The mean curvature flow level set equation and its generalization have numerous applications in boundary problem in material sciences, computational fluid dynamics, image processing, computer vision and many other fields, see $[1,7]$ and references therein. Many papers have been devoted to numerical algorithms for mean curvature flow. These algorithms are related to finite difference methods on uniform grids, threshold dynamics and mathematical morphology using Min/Max operators on game theoretical approach, see [5, 6] for more details.

Recently, there has been a growing interest in the mathematical study of the motion of nonlocal or fractional curvature on Euclidean domains [11, 12].

The main goal of this paper is to propose an adaptation of mean curvature flow level set equation on weighted graphs, using the framework of Partial difference Equations (PdEs) [8]. Our first motivation is to extend the notion of mean curvature to discrete settings and to show the relation between this mean curvature and local and nonlocal forms of curvature in Euclidean domains. The second motivation concerns extending applications of such equation to any discrete data that can be represented by a graph to solve many problems in image and manifold processing. Indeed, many contemporary applications have to deal with large data sets of different types and collected in the form of graphs and networks such as images, videos, meshes, point clouds and images on 3D surfaces. In such situations, any processing and analysis of these data depends on the ability to perform signal or data processing on graphs and networks. Therefore, there is a growing interest in the transposition of signal and image processing tools to process functions defined on graphs. One can quote the recent works on the extension of wavelet on graphs [9]. Similarly, there are many interesting works that aim to mimic and to transpose Partial Differential Equations (PDEs) and variational methods on graphs with applications in image and manifold processing or in machine learning. See $[8,10]$ and references therein for more details.

Short background on PDE level set mean curvature . The level set formulation of the mean curvature flow problem was suggested by Osher and Sethian in [1]. It arises from the front propagation equation and its implementation on the PDEs level set.

Consider the following front propagation problem : we want to find a family $(\Omega)_{t \geq 0}$ of open subsets of $R^{N}$ such that every point $u$ of the boundary $\Gamma_{t}=\partial \Omega_{t}$ (called the front) evolves with a prescribed normal velocity $F$ (which can depend on local or global characteristics). The idea of Osher-Sethian for the level set to analyze moving front is to introduce an auxiliary function $f: \Omega \times[0, T], \Omega \subset R^{n}$ whose 0-level set represents the front $\Gamma_{t}$. We therefore define $u$ such that, for all $t \geq 0, f(u, t)=0$ on $\Gamma_{t}$, $f(u, t)>0$ in $\Omega_{t}$ and $f(u, t)<0$ outside $\Omega_{t}$. Straightforward calculations give the level set PDE on the form $f_{t}(u, t)=F \cdot|\nabla f(u, t)|$. This PDE is complemented with an initial data $\phi_{0}$ which represents the initial front. One can recover $\Gamma_{t}=\{f(u, t)=0\}, \forall t \geqslant 0$. Of course, the specific nature of the level set equation depends on the form of the normal velocity function $F$. For the simple function $F=F(u, t)$, for example, we obtain the Hamilton-Jacobi problem $f_{t}(u, t)=F(u, t) \cdot|\nabla f(u, t)|$ and more general Hamilton-Jacobi equations arise for velocity functions of the form $F=F(n, u, t)$, where $n=n(u, t)=\frac{\nabla f}{|\nabla f|}$ is the 
unit normal to the front at the point $u$ at time $t$. When the normal velocity $F$ also depends on the spatial derivative of the normal vector, we obtain the mean curvature equation:

$$
f_{t}(u, t)=\nabla \cdot\left(\frac{\nabla f(u, t)}{|\nabla f(u, t)|}\right)|\nabla f(u, t)|,
$$

where $f(u, t)$ is an unknown level set function. The quantity $|\nabla f(u, t)|$ is the module of gradient. The level set equation is accompanied by an initial condition $f(u, 0)=$ $f_{0}(u), u \in \Omega$. In the context of image processing, $f_{0}$ corresponds to the given noisy image or to an implicit representation of a front (surface).

Contributions. Our main contributions are as follows. We propose to define the notion of discrete weighted perimeters using a family of discrete gradients on graphs. As in the continuous settings, we introduce the notion of nonlocal curvature as the first variation of the discrete perimeters. We show that our formulation unifies both local and nonlocal notions of the curvature. We also show that our approach can deal with different types of applications including image filtering, images on meshes filtering and 3D surface smoothing.

The transcription of the level set equation on graphs by replacing curvature and gradient leads to a Partial difference Equation. Our proposed numerical scheme leads to a morphological approach alternating dilation and erosion processes.

Paper organization. The rest of this paper is organized as follows. Section 2 presents a general definition of Partial difference Equations on weighted graph. Section 3 presents our new formalism of the Mean Curvature. Section 4 presents some experiments. Finally, Section 5 concludes this paper.

\section{Partial difference Equation on Graphs.}

\subsection{Notations and Definitions.}

We begin briefly by reviewing some basic definitions and operators on weighted graphs. See [2] and references therein for more details.

Let us consider the general situation where any discrete domain can be viewed as a weighted graph. A weighted graph $G=(V, E, w)$ consists in a finite set $V$ of $N$ vertices and in a finite set $E \subseteq V \times V$ of edges. Let $(u, v)$ be the edge that connects vertices $u$ and $v$. An undirected graph is weighted if it is associated with a weight function $w: V \times V \rightarrow[0,1]$. The weight function represents a similarity measure between two vertices of the graph. According to the weight function, the set of edges is defined as : $E=\{(u, v) \mid w(u, v)>0\}$. The degree of a vertex $u$ is defined as $\mu(u)=\sum_{v \sim u} w(u, v)$ where notation $v \sim$ u means that the vertex $v$ is adjacent to $u$. The neighborhood of a vertex $u$ (i.e., the set of vertices adjacent to $u$ ) is denoted $N(u)$. Let $\mathcal{H}(V)$ be the Hilbert space of real valued functions on the vertices of the graph. Each function $f: V \rightarrow \mathbb{R}$ of $\mathcal{H}(V)$ assigns a real value $f(u)$ to each vertex $u \in V$. Similarly, let $\mathcal{H}(E)$ be the Hilbert space of real valued functions defined on the edges of the graph. These two spaces are endowed with the following inner products: $\langle f, h\rangle_{\mathcal{H}(V)}=\sum_{u \in V} f(u) g(u)$ with $f, g \in \mathcal{H}(V)$, and $\langle F, H\rangle_{\mathcal{H}(E)}=\sum_{u \in V} \sum_{v \in V} F(u, v) G(u, v)$ where $F, G \in \mathcal{H}(E)$.

Given a function $f: V \rightarrow \mathbb{R}$, the integral of $f$ is defined as:

$$
\int_{V} f=\sum_{u \in V} f(u)
$$

and its $\mathcal{L}_{p}$ norm is given by:

$$
\begin{aligned}
\|f\|_{p} & =\left(\sum_{u \in V}|f(u)|^{p}\right)^{1 / p}, 1 \leqslant p<\infty . \\
\|f\|_{\infty} & =\max _{u \in V}(|f(u)|), p=\infty .
\end{aligned}
$$

\subsection{Difference, Divergence and Discrete Gradients on Graphs}

Let us fix a weighted graph $G=(V, E, w)$. The difference operator $\mathcal{G}_{w}: \mathcal{H}(V) \rightarrow \mathcal{H}(E)$ is given for all $f \in \mathcal{H}(V)$ and $(u, v) \in E$ by: $\left(\mathcal{G}_{w} f\right)(u, v)=\gamma(u, v)(f(v)-f(u))$, with $\gamma: \mathbb{R}^{+} \rightarrow \mathbb{R}^{+}$ and $\gamma(u, v)=\gamma(w(u, v))$ with $\gamma(0)=0$.

The directional derivative (or edge derivative) of a function $f$ at a vertex $v$ along an edge $e=u v$, is defined as $\partial_{v} f_{u}=\left(\mathcal{G}_{w} f\right)(u, v)=\gamma(u, v)(f(v)-f(u))$.

The adjoint operator of the difference operator, denoted by $\mathcal{G}_{w}^{*}: \mathcal{H}(E) \rightarrow \mathcal{H}(V)$, is defined by $\left\langle\mathcal{G}_{w} f, H\right\rangle_{\mathcal{H}(E)}=\left\langle f, \mathcal{G}_{w}^{*} H\right\rangle_{\mathcal{H}(V)}$, with $f \in \mathcal{H}(V)$ and $H \in \mathcal{H}(E)$. Using the definitions of the inner products in $\mathcal{H}(V)$ and $\mathcal{H}(E)$ and the definition of the difference operator, we obtain easily the expression $\mathcal{G}_{w}^{*}$ at a vertex $u:\left(\mathcal{G}_{w}^{*} H\right)(u)=\sum_{v \sim u} \gamma(u, v)(H(v, u)-H(u, v))$.

The divergence operator, defined by $d i v_{w}=-\mathcal{G}_{w}^{*}$, measures the net outflow of a function in $\mathcal{H}(E)$ at each vertex of $V$.

Two weighted directional difference operators can be defined. The weighted directional external and internal difference operators are respectively:

$$
\begin{aligned}
& \left(\partial_{v}^{+} f\right)(u)=\gamma(u, v)(f(v)-f(u))^{+} \text {and } \\
& \left(\partial_{v}^{-} f\right)(u)=\gamma(u, v)(f(v)-f(u))^{-},
\end{aligned}
$$


with $(x)^{+}=\max (0, x)$ and $(x)^{-}=-\min (0, x)$.

The weighted gradient of a function $f \in \mathcal{H}(\mathbf{V})$ at vertex $u$ is the vector of all edge directional derivatives:

$$
\left(\nabla_{w} f\right)(u)=\left(\partial_{v} f(u)\right)_{v \in V}^{T}
$$

Two discrete formulations of weighted morphological gradients on graphs are defined. The weighted external $\nabla_{w}^{+}$ and the internal $\nabla_{w}^{-}$gradient operators are respectively:

$$
\begin{aligned}
& \left(\nabla_{w}^{+} f\right)(u)=\left(\partial_{v}^{+} f(u)\right)_{v \in V}^{T}, \\
& \left(\nabla_{w}^{-} f\right)(u)=\left(\partial_{v}^{-} f(u)\right)_{v \in V}^{T} .
\end{aligned}
$$

To define a notion of regularity of a function $f$ around a vertex $u$, we can consider different norms of gradients as follows:

$$
\left\|\left(\nabla_{w}^{ \pm} f\right)(u)\right\|_{p}=\left[\sum_{v \sim u} \gamma(u, v)^{p}\left((f(v)-f(u))^{ \pm}\right)^{p}\right]^{1 / p}
$$

$$
\left.\left\|\left(\nabla_{w}^{ \pm} f\right)(u)\right\|_{\infty}=\max _{v \sim u}\left(\gamma(u, v)\left|(f(v)-f(u))^{ \pm}\right|\right)\right) .
$$

$\nabla_{w}^{ \pm}$refers to both external and internal gradient (with respect to the sign). These gradients have the following property:

$$
\left\|\left(\nabla_{w} f\right)(u)\right\|_{p}^{p}=\left\|\left(\nabla_{w}^{+} f\right)(u)\right\|_{p}^{p}+\left\|\left(\nabla_{w}^{-} f\right)(u)\right\|_{p}^{p} .
$$

Moreover, with a constant weight function and $p=\infty$, equation (8) recovers the usual expression of algebraic morphological external and internal gradients.

These gradients are used in [2] to adapt the well-known Eikonal equation on discrete domains defined as:

$$
\frac{\partial_{f}}{\partial_{t}}(u, t)=\mu(u)\|(\nabla f)(u, t)\|_{p}, \mu(u) \in \mathbb{R},
$$

in their settings, this adaptation is given by:

$$
\frac{\partial_{f}}{\partial_{t}}(u, t)=\mu^{+}(u)\left\|\left(\nabla_{w}^{+} f\right)(u)\right\|_{p}-\mu^{-}(u)\left\|\left(\nabla_{w}^{-} f\right)(u)\right\|_{p}
$$

This equation summarizes the dilation and erosion processes. When $\mu>0$, then the external gradient is used and this equation corresponds to a dilation. When $\mu<0$, this equation corresponds to an erosion.

\section{Mean Curvature on Graph}

In this section, we present our new definition of mean curvature on graph by introducing nonlocal perimeters on graph. We define the mean curvature as the first variation of these perimeters. We will show that the transcription of the mean curvature equation (1) using our definition and the morphological gradients leads to a difference equation solved by a simple and iterative digital algorithm involving morphological erosion and dilatation on graph.

\subsection{Nonlocal Perimeters and Co-Area Formula on Graph}

Let $\mathcal{A}$ be a set of connected vertices with $\mathcal{A} \subset V$. We denote $\partial^{+} \mathcal{A}=\left\{u \in \mathcal{A}^{c} \mid \exists v \in \mathcal{A}, v \sim u\right\}$ be the outer vertex boundary, let $\partial^{-} \mathcal{A}=\left\{u \in \mathcal{A} \mid \exists v \in \mathcal{A}^{c}, v \sim u\right\}$ be the inner vertex boundary where $\mathcal{A}^{c}$ is the complement of $\mathcal{A}$. Let $\partial_{v} \mathcal{A}=\partial^{+} \mathcal{A} \cup \partial^{-} \mathcal{A}$ be the symmetric vertex boundary. Note that $\partial^{+} \mathcal{A}=\partial^{-}\left(\mathcal{A}^{c}\right), \partial^{-}(\mathcal{A})=\partial^{+}\left(\mathcal{A}^{c}\right)$ and $\partial(\mathcal{A})=\partial\left(\mathcal{A}^{c}\right)$. We define the edge boundary $\partial_{E} \mathcal{A}=$ $\left\{(u, v) \in E, u \in \mathcal{A}, v \in \mathcal{A}^{c}\right\}$. Let $\chi$ be an indicator function with :

$$
\chi_{\mathcal{A}}(u)= \begin{cases}1 & \text { if } u \in \mathcal{A} \\ 0 & \text { otherwise. }\end{cases}
$$

Proposition. Let $1 \leq p<\infty$ and $\mathcal{A} \subset V$.

$$
\left\|\nabla_{w}^{+} \chi_{\mathcal{A}}(u)\right\|_{p}=\left(\sum_{v \in \mathcal{A}}(\gamma(u, v))^{p}\right)^{1 / p} \chi_{\partial^{+} \mathcal{A}}(u)
$$

$$
\begin{aligned}
& \left\|\nabla_{w}^{-} \chi_{\mathcal{A}}(u)\right\|_{p}=\left(\sum_{v \in \mathcal{A}^{c}}(\gamma(u, v))^{p}\right)^{1 / p} \chi_{\partial^{-} \mathcal{A}}(u) . \\
& \left\|\nabla_{w} \chi_{\mathcal{A}}(u)\right\|_{p}=\left\|\nabla_{w}^{+} \chi_{\mathcal{A}}(u)\right\|_{p}+\left\|\nabla_{w}^{-} \chi_{\mathcal{A}}(u)\right\|_{p} .
\end{aligned}
$$

The above equations were obtained by replacing the different variables $\left(f\right.$ by $\chi_{\mathcal{A}}$ ) in equations $(6)(8)$.

We see that for $u \in \mathcal{A},\left\|\nabla_{w}^{+} \chi_{\mathcal{A}}(u)\right\|_{p}$ is just the weighted number of neighbors of the vertices $u$ that belongs to $\mathcal{A}^{c}$ (equivalently the weighted numbers of the out-boundary edges between $u$ and $\mathcal{A}^{c}$ ), while $\left\|\nabla_{w}^{-} \chi_{\mathcal{A}}(u)\right\|_{p}$ is the weighted number of in-boundary edges between neighbors of the vertices $u$ that belongs to $\mathcal{A}^{c}$, and $\left\|\nabla_{w} \chi_{\mathcal{A}}(u)\right\|_{p}$ is the weighted number of in-boundary and out-boundary edges.

Based on the previous definitions, let us define a family of perimeters on graph. 
Definition. For $0<p<\infty$ and $\mathcal{A} \subset V$, the perimeter of $\mathcal{A}$ is defined as:

$$
\begin{aligned}
& \operatorname{Per}_{w, p}^{+}(\mathcal{A})=1 / 2 p \sum_{u \in V}\left\|\nabla_{w}^{+} \chi_{\mathcal{A}}(u)\right\|_{p}=J_{w, p}^{+}\left(\chi_{\mathcal{A}}(u)\right) . \\
& \operatorname{Per}_{w, p}^{-}(\mathcal{A})=1 / 2 p \sum_{u \in V}\left\|\nabla_{w}^{-} \chi_{\mathcal{A}}(u)\right\|_{p}=J_{w, p}^{-}\left(\chi_{\mathcal{A}}(u)\right) . \\
& \operatorname{Per}_{w, p}(\mathcal{A})=1 / p \sum_{u \in V}\left\|\nabla_{w} \chi_{\mathcal{A}}(u)\right\|_{p}=J_{w, p}\left(\chi_{\mathcal{A}}(u)\right) .
\end{aligned}
$$

Where $J_{w, p}$ is a regularization functional and defined as:

$$
\begin{aligned}
& J_{w, p}^{+} f(u)=1 / 2 p \sum_{u \in V}\left\|\left(\nabla_{w}^{+} f\right)(u)\right\|_{p} . \\
& J_{w, p}^{-} f(u)=1 / 2 p \sum_{u \in V}\left\|\left(\nabla_{w}^{-} f\right)(u)\right\|_{p} . \\
& J_{w, p} f(u)=1 / p \sum_{u \in V}\left\|\left(\nabla_{w} f\right)(u)\right\|_{p} .
\end{aligned}
$$

Remark. If we consider the case where $p=1$, it is easy to show that

$\operatorname{Per}_{w, 1}^{+}(\mathcal{A})=\operatorname{Per}_{w, 1}^{-}(\mathcal{A})$ and $\operatorname{Per}_{w, 1}(\mathcal{A})=$ $2 \operatorname{Per}_{w, 1}^{+}(\mathcal{A})=\sum_{u \in V}\left\|\nabla_{w} \chi_{\mathcal{A}}\right\|_{1}$, and

$$
\operatorname{Per}_{w, 1}^{+}(\mathcal{A})=\sum_{u \in \mathcal{A}^{c}} \sum_{v \in \mathcal{A}} \gamma(u, v)
$$

which is the definition of the graph cut formulation.

As in the continuous case where the perimeter is linked to the total variation via co-area formula, we will show that our proposed perimeters cover this property.

A key property of the gradient in this case is called co-area formula. This is an extension of some properties of the total variation on graph.

Proposition. For any function $f: V \rightarrow R$

$$
D f=\int_{-\infty}^{+\infty} D \chi_{\{f>t\}} d t
$$

where $\mathrm{D}$ denotes either one of $\left|\nabla_{w}\right|_{1},\left|\nabla_{w}\right|_{1}^{ \pm},\left|\nabla_{w}\right|_{\infty}^{ \pm}$.

$\mathrm{D}$ are the functions of the form $\sum_{u \in V} \gamma(u, v)(f(v)-$ $f(u))^{ \pm}$and $\sum_{u \in V}(\gamma(u, v)|f(v)-f(u)|$, where the weights $\gamma(u, v)$ are non negative.
Proof. This proposition just follows easily from $|a-b|=$ $\int_{-\infty}^{+\infty}\left|\chi_{\{a>t\}}-\chi_{\{b>t\}}\right| d t$, and $(a-b)^{ \pm}=\int_{-\infty}^{+\infty}\left(\chi_{\{a>t\}}-\right.$ $\left.\chi_{\{b>t\}}\right)^{ \pm} d t$, where $a$ and $b \in R$.

The above proposition allows to recover the following definitions which can be used to relax many problem of optimization involving the discrete perimeters:

$$
\begin{aligned}
J_{w, 1}^{+}(f) & =\int_{-\infty}^{+\infty} J_{w, 1}^{+}\left(\chi_{\{f(u)>t\}}\right) d t \\
& =\int_{-\infty}^{+\infty} \operatorname{Per}_{w, 1}^{+}\left(\chi_{\{f(u)>t\}}\right) d t . \\
J_{w, 1}^{-}(f) & =\int_{-\infty}^{+\infty} J_{w, 1}^{-}\left(\chi_{\{f(u)>t\}}\right) d t \\
& =\int_{-\infty}^{+\infty} \operatorname{Per}_{w, 1}^{-}\left(\chi_{\{f(u)>t\}}\right) d t . \\
J_{w, 1}(f) & =\int_{-\infty}^{+\infty} J_{w, 1}\left(\chi_{\{f(u)>t\}}\right) d t \\
& =\int_{-\infty}^{+\infty} \operatorname{Per}_{w, 1}\left(\chi_{\{f(u)>t\}}\right) d t .
\end{aligned}
$$

As in the continuous domain, we will define the mean curvature as the first variation of the perimeters that we just defined.

Definition. Let $u_{0} \in \partial \mathcal{A}=\partial^{+} \mathcal{A} \cup \partial^{-} \mathcal{A}$.

For $u_{0} \in \partial^{+} \mathcal{A}$, the mean curvature of $u_{0}$ is defined as:

$$
\mathcal{K}_{w}^{+}\left(u_{0}, \mathcal{A}\right)=\frac{\operatorname{Per}_{w, 1}^{+}\left(\mathcal{A} \cup\left\{u_{0}\right\}\right)-P e r_{w, 1}^{+}(\mathcal{A})}{\mu\left(u_{0}\right)},
$$

where $\mu\left(u_{0}\right)$ define the degree of a vertex $u_{0}$. And for $u_{0} \in$ $\partial^{-} \mathcal{A}$, the mean curvature of $u_{0}$ is defined as:

$$
\mathcal{K}_{w}^{-}\left(u_{0}, \mathcal{A}\right)=\frac{\operatorname{Per}_{w, 1}^{-}(\mathcal{A})-P e r_{w, 1}^{-}\left(\mathcal{A}-\left\{u_{0}\right\}\right)}{\mu\left(u_{0}\right)} .
$$

By replacing the variables $\left(\mathcal{A}\right.$ by $\left.\mathcal{A} \cup\left\{u_{0}\right\}\right)$ in equation (14), it can be rewritten as:

$$
\begin{aligned}
\operatorname{Per}_{w, 1}^{+}\left(\mathcal{A} \cup\left\{u_{0}\right\}\right) & =\sum_{v \in\left(\left\{\mathcal{A} \cup\left\{u_{0}\right\}\right)^{c}\right.} \sum_{v \in\left(\left\{\mathcal{A} \cup\left\{u_{0}\right\}\right)\right.} \gamma(u, v) \\
& =\sum_{v \in\left(\mathcal{A}^{c}-\left\{u_{0}\right\}\right)} \sum_{v \in\left(\mathcal{A} \cup\left\{u_{0}\right\}\right)} \gamma(u, v) .
\end{aligned}
$$


It is easy to show that:

$$
\begin{array}{r}
\operatorname{Per}_{w, 1}^{+}\left(\mathcal{A} \cup\left\{u_{0}\right\}\right)-\operatorname{Per}_{w, 1}^{+}(\mathcal{A})= \\
\sum_{v \in \mathcal{A}^{c}} \gamma\left(u_{0}, v\right)-\sum_{v \in \mathcal{A}} \gamma\left(u_{0}, v\right),
\end{array}
$$

then the mean curvature $\mathcal{K}_{w}^{+}$of $u_{0}$ is rewritten as:

$\mathcal{K}_{w}^{+}\left(u_{0}, \mathcal{A}\right)=\frac{\sum_{v \in \mathcal{A}^{c}} \gamma\left(u_{0}, v\right)-\sum_{v \in \mathcal{A}} \gamma\left(u_{0}, v\right)}{\mu\left(u_{0}\right)}$.

Similarly, the mean curvature $\mathcal{K}_{w}^{-}$of $u_{0}$ is rewritten as:

$$
\mathcal{K}_{w}^{-}\left(u_{0}, \mathcal{A}\right)=\frac{\sum_{v \in \mathcal{A}^{c}} \gamma\left(u_{0}, v\right)-\sum_{v \in \mathcal{A}} \gamma\left(u_{0}, v\right)}{\mu\left(u_{0}\right)} .
$$

Then, for $u_{0} \in \partial \mathcal{A}$, the mean curvature is defined as:

$$
\mathcal{K}_{w}\left(u_{0}, \mathcal{A}\right)=\frac{\sum_{v \in \mathcal{A}^{c}} \gamma\left(u_{0}, v\right)-\sum_{v \in \mathcal{A}} \gamma\left(u_{0}, v\right)}{\mu\left(u_{0}\right)} .
$$

Based on this definitions, we can extend the notion of curvature to any function $f$ defined on a graph.

Let $f: V \rightarrow \mathbb{R}$ and $u \in V$. The mean curvature $k_{w}$ of $\mathrm{f}$ at $u$ on a graph is defined as:

$$
\begin{aligned}
& \mathcal{K}_{w}(u, f)=\mathcal{K}_{w}(u,\{f(v) \geq f(u)\})= \\
& \frac{\sum_{f(v)-f(u) \geq 0} \gamma(u, v)-\sum_{f(v)-f(u)<0} \gamma(u, v)}{\mu(u)} \\
& =\frac{\sum_{u \in V} \gamma(u, v) \operatorname{sign}(f(v)-f(u))}{\mu(u)},
\end{aligned}
$$

with $\operatorname{sign}(r)=\left\{\begin{array}{l}1 \quad \text { if } r \geq 0 \\ -1 \quad \text { otherwise. }\end{array}\right.$

\subsection{Connection with Nonlocal Mean Curvature and Euclidean Graph}

We will show that our definition of mean curvature is linked to the notion of fractional mean curvature introduced in [11]. The notion of the fractional perimeter (s-perimeter) and the corresponding minimization problem were introduced in [12]. The s-perimeter of $\mathcal{A} \subset \mathbb{R}^{\mathrm{n}}$ is defined as:

$$
\operatorname{Per}_{s}(\mathcal{A})=c_{n} \int_{\mathcal{A}} \int_{\mathcal{A}^{c}} \frac{1}{|x-y|^{n+s}} d x d y
$$

where $x$ and $y \in \mathcal{A}$ and $c_{n}$ is a normalization constant and |.| is the euclidean norm on $\mathbb{R}^{\mathrm{n}}$.
The main idea of the s-perimeter is that any point inside $\mathcal{A}$ interact with any point outside $\mathcal{A}$. The continuous fractional curvature is defined formally as the first variation of these s-perimeter as follow:

$$
\begin{array}{r}
\mathcal{K}(x, \mathcal{A})=c_{n} \int_{\mathcal{A}} \frac{\chi_{\mathcal{A}}(y)-\chi_{\mathcal{A}^{c}}(y)}{|x-y|^{n+s}} d y= \\
c_{n} \int_{\mathcal{A}} \frac{1}{|x-y|^{n+s}} d y-c_{n} \int_{\mathcal{A}^{c}} \frac{1}{|x-y|^{n+s}} d y .
\end{array}
$$

The above equation can be interpreted as a continuous version of our proposed definition. Let us consider an euclidean graph $G=(V, E, w)$ with $V=\mathbb{R}^{\mathrm{n}}, \mathcal{A} \subset V$, $E=\{(x, y) \in V \times V / w(x, y)>0\}, \mathcal{A} \subset \mathbb{R}^{\mathrm{n}}$.

For $w(x, y)=\left\{\begin{array}{l}\frac{1}{|x-y|^{n+s}} \text { with } 0<s<1 \\ 0 \quad \text { otherwise }\end{array}\right.$

our mean curvature is defined as:

$$
\mathcal{K}_{w}(x, \mathcal{A})=\frac{\sum_{y \in \mathcal{A}^{c}} w(x, y)-\sum_{y \in \mathcal{A}} w(x, y)}{\mu(x)} .
$$

One can see that the above equation corresponds to the continuous version of equation (36).

\subsection{Numerical Scheme}

Based on the definition of our mean curvature and the transcription of equation (1), our formulation can be expressed for the case of $L_{\infty}$ norm as follow:

$\left\{\begin{array}{l}\frac{\partial f}{\partial t}(u)=\mathcal{K}_{w}^{+}(f(u))\left\|\left(\nabla_{w}^{+} f\right)(u)\right\|_{\infty}-\mathcal{K}_{w}^{-}(f(u))\left\|\left(\nabla_{w}^{-} f\right)(u)\right\|_{\infty} \\ f(u, 0)=f_{0}(u) .\end{array}\right.$

where $\mathcal{K}_{w}^{+}(x)=\left(\mathcal{K}_{w}(x)\right)^{+}$and $\mathcal{K}_{w}^{-}(x)=\left(\mathcal{K}_{w}(x)\right)^{-}$.

Now, let us show that this iterative equation corresponds to alternate dilation and erosion (type filter) processes depending on the sign of $\mathcal{K}_{w}$. We introduce two operators, nonlocal dilation (NLD) and nonlocal erosion (NLE) that are defined respectively as:

$$
N L D(f(u))=f(u)+\left\|\left(\nabla_{w}^{+} f\right)(u)\right\|_{\infty}
$$

$$
N L E(f(u))=f(u)-\left\|\left(\nabla_{w}^{-} f\right)(u)\right\|_{\infty}
$$

The time variable is discretized using the explicit Euler method as:

$$
\frac{\partial f}{\partial t}(u)=\frac{f^{n+1}(u)-f^{n}(u)}{\Delta t}
$$


where $f^{n}(u)=f(u, n \Delta t)$ and equation (38) can be rewritten as the following iterative equation:

$$
\begin{aligned}
f^{n+1}(u) & =f^{n}(u)\left(1-\Delta t\left[\mathcal{K}_{w}^{+}\left(f^{n}(u)\right)+\mathcal{K}_{w}^{-}\left(f^{n}(u)\right)\right]\right) \\
& +\Delta t \mathcal{K}_{w}^{+}\left(f^{n}(u)\right) N L D\left(f^{n}(u)\right) \\
& +\Delta t \mathcal{K}_{w}^{-}\left(f^{n}(u)\right) N L E\left(f^{n}(u)\right)
\end{aligned}
$$

To solve this dilation and erosion processes, in contrast to the PDEs case, no spatial discretization is needed thanks to derivatives directly expressed in a discrete form.

In the case where $\mathcal{K}_{w} \geq 0$, equation (42) summarizes the averaging between the initial function and the NLD operator and can be rewritten as:

$$
\begin{aligned}
f^{n+1}(u) & =f^{n}(u)\left(1-\Delta t \mathcal{K}_{w}^{+}\left(f^{n}(u)\right)\right) \\
& +\Delta t \mathcal{K}_{w}^{+}\left(f^{n}(u)\right) N L D\left(f^{n}(u)\right) .
\end{aligned}
$$

In the case where $1-\Delta t \mathcal{K}_{w}^{+}=0$, equation (43) can be interpreted as an iterative nonlocal dilation (NLD) process.

Similarly, in the case where $\mathcal{K}_{w}<0$, equation (42) summarizes the averaging between the initial function and NLE operator, and can be interpreted as an iterative NLE process.

In the case where $\mathcal{K}_{w} \neq 0$ and $1-\Delta t\left|\mathcal{K}_{w}\right| \geq 0$, equation (43) summarizes an average filtering process.

At each step of this algorithm, the new value at vertex $u$ depends only on its value at step $n$ and the existing values in its neighborhood.

\section{Applications}

The proposed formulation of mean curvature flow equation can be used to process any function defined on vertices of a graph or on any arbitrary discrete domain.

This section illustrates the potentialities of our formulation through examples of image filtering, image on surface filtering and 3D surfaces smoothing. Different graph structures and weight functions are also used to show the flexibility of our approach. The objective of the following experiments is not to solve a particular application, they only illustrate the potential and the behavior of our mean curvature definition formulation.

Weighted graph construction. Any discrete domain can be represented by a weighted graph where functions of $\mathcal{H}(V)$ represents the data to process. In the general case, an unorganized set of points $V \subset \mathbb{R}^{\mathrm{n}}$ can be seen as a function $f^{0}: V \subset R^{n} \rightarrow R^{n}$. Then, constructing a graph from this data consists in defining the set of edges $E$ by modeling the neighborhood. It is based on a similarity relationship between data with a pairwise distance measure $d: V \times V \rightarrow R^{+}$. In this paper, we focus on two particular graphs: the grid graphs and the k-nearest neighbors graphs. Grid graphs which are the natural structure to describe an

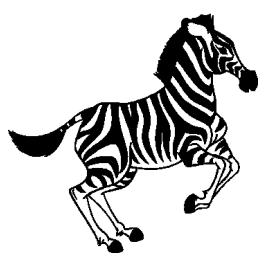

(a)

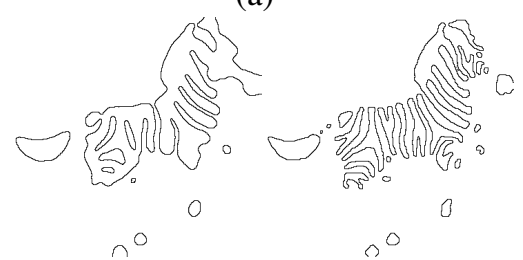

(b)

(c)

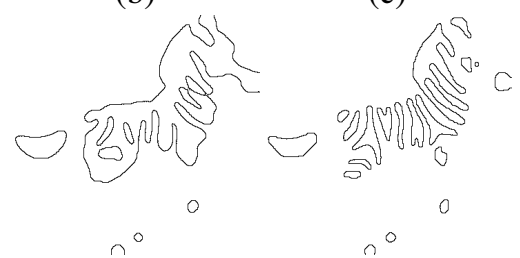

(d)

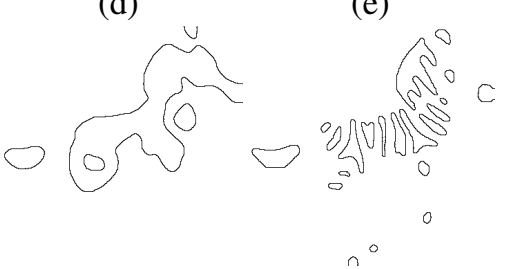

(f)

(g)

Figure 1. Mean Curvature evolution of a zebra curve. (a) presents the initial zebra image. (b, d, f) present the curve motion by mean curvature on a local structure graph after 5, 10 and 20 iterations. (c, e, g) present the curve motion by mean curvature on a nonlocal graph structure after 5,10 and 20 iterations.

image with a graph. Each pixel is connected by an edge to its adjacent pixels. The $\mathrm{k}$ nearest neighbors graph, noted $\mathrm{k}-\mathrm{NNG}$ is a weighted graph where each vertex $u \in V$ is connected to its $\mathrm{k}$ nearest neighbors which have the smallest distance measure towards $u$ according to function $d$.

Then, the weight function $w$ can be defined using usual similarity functions depending on application and graph topology (the weight function is defined as the Gaussian kernel), and satisfies:

$$
w(u, v)=\left\{\begin{array}{l}
g(u, v) \quad \text { if }(u, v) \in E \\
0 \quad \text { otherwise. }
\end{array}\right.
$$

where $g$ is one of the following similarity functions:

$$
\begin{aligned}
& g_{0}(u, v)=1 . \\
& g_{1}(u, v)=e^{\left(-d\left(f^{0}(u), f^{0}(v)\right) / \sigma^{2}\right)} \text { with } \sigma>0 .
\end{aligned}
$$

Mean curvature for shape evolution. Let $f_{0}$ be a level set function that represents our initial data where $f_{0}=$ $\chi_{\Omega_{O}}-\chi_{\Omega_{0}^{c}}$, with $\chi: V \rightarrow\{0,1\}$ is the indicator function and $\Omega_{0}^{c}$ is the complement of $\Omega_{0}$. In other words, 
$f_{0}$ equals 1 in $\Omega_{0}$ and -1 on its complementary. First in figure 1, we show the evolution of the zebra curve under the effect of mean curvature flow using local and nonlocal graph structures. The first column presents the evolution of the zebra curve using a local graph (i.e. 4-adjacency grid graph) with a constant weight function $w(u, v)=1$ at different steps. Second column presents the same results but on a nonlocal graph. In this example, the graph is constructed as a 16-adjacency grid graph with the weight function $w(u, v)=e^{\left(-\|u-v\| / \sigma^{2}\right)}$ and $\sigma=10$. One can see that the motion of the curve with a nonlocal graph preserve the global form of the initial zebra curve.

Mean curvature for image filtering. In this paragraph, we illustrate the behavior of our approach to perform noisy images filtering. Let $f_{0}: V \rightarrow \mathbb{R}^{\mathrm{n}}$ be a level function set that represents initial data. Figure 2 presents a comparison between the effect of filtering of a noisy image using mean curvature flow on local and nonlocal graph structures. The first line presents results obtained on local graph. The second line presents results obtained on nonlocal graph. In this example, we construct a 16-adjacency grid graph with $w(u, v)=e^{\left(-d(\mathcal{F}(u), \mathcal{F}(v)) / \sigma^{2}\right)}$ and $\sigma=20$, where $\mathcal{F}: V \subset \mathbb{R}^{\mathrm{n}}$ associates a patch of pixels to every vertices (this to better describe texture informations). Both lines presents results at different steps of mean curvature flow. Similarly to the previous example, one can remark that the nonlocal structure better preserve image details and textures. Figure 3 presents another filtering example on noisy images using nonlocal graph structure.

Application to images on surfaces filtering. In this paragraph, we illustrate the adaptivity of our approach to perform noisy image on surfaces filtering. The approach is the same that for image on regular grid, but with a different graph topology. Figure 4 presents the filtering of a image on a 3D surface (or mesh). The first line shows the initial model and the second one shows the filtering result. In this example, the graph was constructed using the mesh structure.

Application to 3D surface smoothing. Another application that illustrates the adaptivity of our approach is the $3 \mathrm{D}$ surfaces smoothing. Figure 5 presents a $3 \mathrm{D}$ noisy surfaces smoothing using mean curvature flow and the fast surface reconstruction method presented in [3]. In this example, the graph is a k-NNG graph constructed from the set of initial points and numerous additional points as described in [3]. The weight function is given by $w(u, v)=e^{\left(-d\left(f^{0}(u), f^{0}(v)\right) / \sigma^{2}\right)}$ with $\sigma=10$. Similarly to the first example, the level set function $f_{0}$ is defined as : $f_{0}=\chi_{\Omega_{O}}-\chi_{\Omega_{0}^{c}}$.

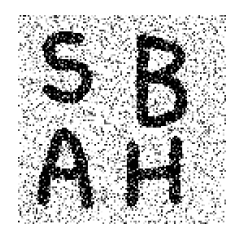

(a)

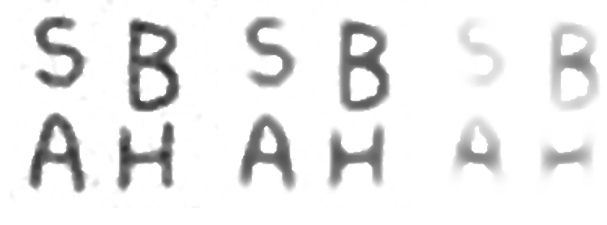

(b)

(c)

(d)

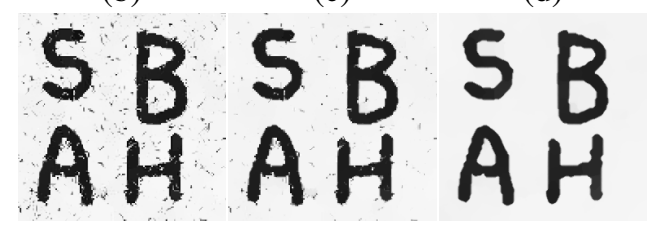

(e)

(f)

(g)

Figure 2. Noisy image filtering. (a) presents the initial image, $(b, c, d)$ present the filtering using mean curvature on a local graph structure after 5, 10 and 20 iterations.(e,f,g) present the filtering using mean curvature on a nonlocal graph structure after 5, 10 and 20 iterations.

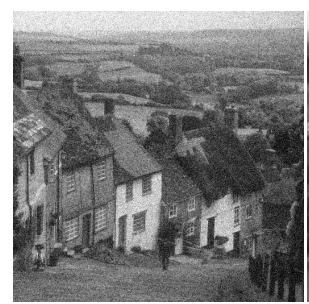

(a)

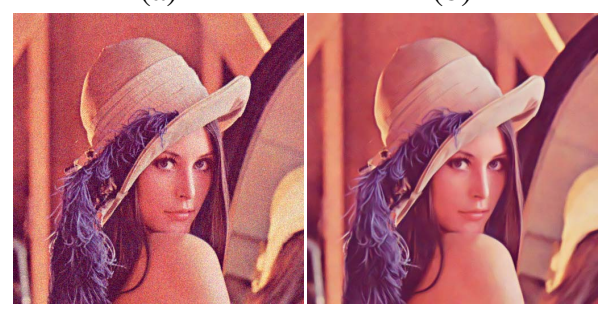

(c)

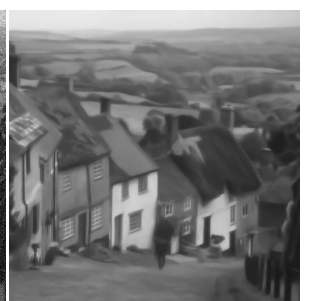

(b)
Figure 3. Noisy Image filtering. (a,b) present the GoldHill noisy image and the filtered one after 30 iterations with $\sigma=20$, (c,d) present the noisy colored Lena image and the filtered one after 30 iterations with $\sigma=20$.

\section{Conclusion}

In this paper, we have introduced an adaptation of the mean curvature on weighted graphs as the first variation of perimeters, based on PdEs and using a framework of discrete operators. Experimental results have shown the potentiality of the proposed formulation of mean curvature level 


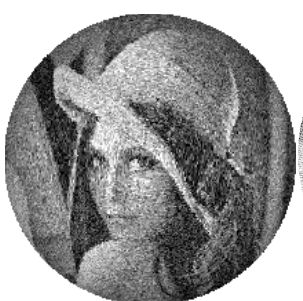

(a)

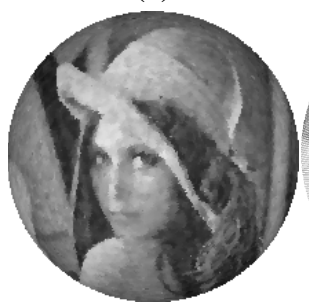

(c)

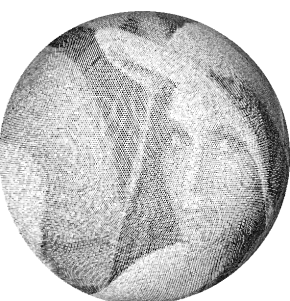

(b)

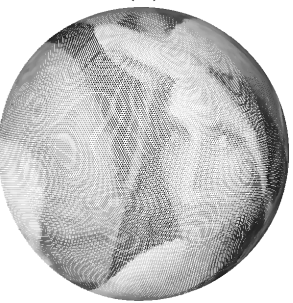

(d)
Figure 4. Image on mesh filtering. (a) presents the initial noisy image on surface, (b) presents a zoom on the point clouds of the initial model, (c) presents the filtered model by mean curvature after 30 iterations with $\sigma=20$, (d) presents a zoom on the point clouds of the filtered model.

sets and its adaptivity to graphs of arbitrary topology.

\section{References}

[1] J. A. Sethian Level Set Methods and Fast Marching Methods.: Some Fine Journal, Cambridge University Press, 1999.

[2] X. Desquesnes and al. : Eikonal equation adaptation on weighted graphs: fast geometric diffusion process for local and non-local image and data processing.. Journal of Mathematical Imaging and Vision.

[3] A. EL Chakik and al.: Fast 3D surface reconstruction from point clouds using graph-based fronts propagation. ACCV, 2012, Part IV, LNCS 7727, p. 309.

[4] X. Desquesnes and al.: Efficient Algorithms for Image and high Dimensional Data Processing Using Eikonal Equation on Graphs. Some Fine Journal, International Symposium on Visual Computing, Vol. LNCS 6454, pp. 647-658, 2010.

[5] L. Alvarez and al. : Axioms and fundamental equations of image processing. Arch. Rational Mech. Anal., 123(3):199257, 1993.

[6] Rudd Matthew : Game-theoretic schemes for generalized curvature flows in the plane. Electronic Journal of Differential Equations, 2007.

[7] F. Catte and al. : A morphological scheme for mean curvature motion and applications to anisotropic diffusion and motion of level sets. SIAM J. Numer. Anal., 32 (1995), pp. 18951909.

[8] A. Elmoataz and al.: Nonlocal discrete regularization on weighted graphs: A framework for image and manifold processing. IEEE Trans. Image Process. 17 (7), 10471060 (2008).

[9] D.K. Hammond and al. : Wavelets on graphs via spectral graph theory. Appl. Comp. Harmonic Analysis, vol. 30, no. 2, pp. 129-150, 2011.

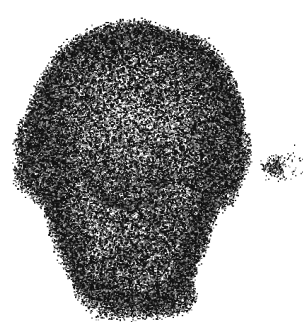

(a)

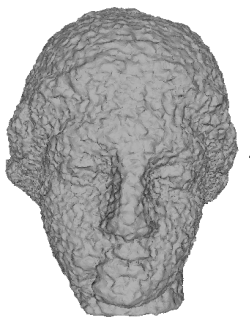

(c)

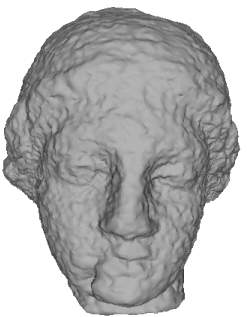

(e)

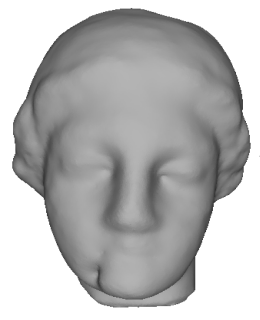

(g)

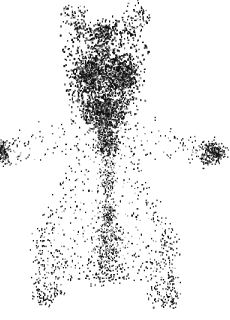

(b)

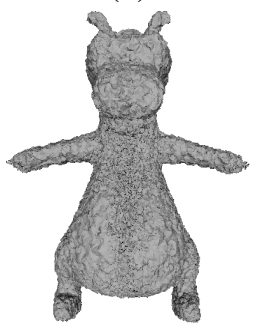

(d)

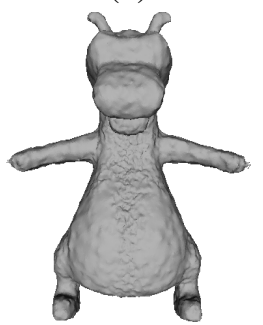

(f)

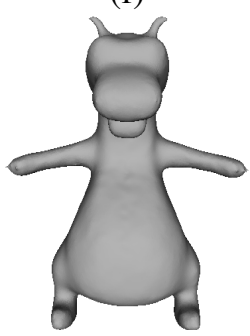

(h)
Figure 5. 3D Surfaces smoothing. (a, c, e, g) present respectively the initial 3D noisy Venus point clouds, the reconstructed surface, the Venus surface after 5 iterations using our mean curvature on nonlocal graph structure, the Venus surface after 15 iterations. $(\mathrm{b}, \mathrm{d}, \mathrm{f}, \mathrm{h})$ present respectively the initial 3D noisy Dragon point clouds, the reconstructed surface, the Dragon surface after 5 iterations using our mean curvature on nonlocal graph structure, the Dragon surface after 15 iterations.

[10] V.-T. Ta and al. : Partial difference equations over graphs: Morphological processing of arbitrary discrete data. in Proceedings of ECCV, 2008, vol. LNCS 5304, pp. 668680.

[11] C. Imbert : Level set approach for fractional mean curvature flows. Interfaces Free Bound., 11 (2009), pp. 153-176.

[12] A. Chambolle and al. : A Nonlocal Mean Curvature Flow and Its Semi-implicit Time-Discrete Approximation. SIAM J. Math. Analysis 44(6): 4048-4077 (2012). 